\title{
Deadline Maximum Signal to Interference Ratio Scheduling Algorithm for WiMAX
}

\author{
Rabee Mustapha A. Abuteir \\ Computer Engineering Department \\ Faculty of Engineering \\ Gaza, Palestine
}

\author{
Aiman A. Abu Samra \\ Computer Engineering Department \\ Faculty of Engineering \\ Gaza, Palestine
}

\begin{abstract}
The IEEE 802.16 standard, which is called Worldwide Interoperability for Microwave Access (WiMAX) is a low cost solution for Internet access in metropolitan and rural areas; it provides both high throughput and large coverage broadband wireless access. Although it defines five service level classes to support real-time and bandwidth demanding applications, each class is associated with a set of Quality of Service (QoS) parameters. However, the standard does not specify which scheduling algorithm should be used to serve packets. Due to the wireless channel variability, scheduling mechanisms widely studied for wired networks are not suitable for IEEE 802.16 networks. In this paper, we introduce Deadline maximum Signal to Interference Ratio scheduler, which makes bandwidth allocation decisions based on information about the channel quality and deadline. Simulation results show the proposed approach gains more delivering packet and decreases the average end to end delay and improve the fairness index.
\end{abstract}

\section{General Terms}

Wireless, Broadband, Algorithms.

\section{Keywords}

IEEE 802.16, WiMAX, scheduling, QoS, SNR, ns-2.

\section{INTRODUCTION}

The IEEE 802.16 standard, widely known as Worldwide Interoperability for Microwave Access (WiMAX) which developed for Broadband Wireless Access (BWA). The standard defines Two basic operational modes mesh mode and Point to Multiple Point (PMP) mode. In mesh mode, Subscriber Stations (SSs) communicate directly each other. In PMP mode the Base Station (BS) is connected to various Subscriber Stations and they communicate only over a Base Station, our focus is only on PMP network configuration [1-2]

WIMAX provides the details of physical (PHY) layer and Media Access Control (MAC) sublayers of an advanced wireless communication system which aims to build a cost effective, high speed data rate, last mile wireless access, multi-service network and QoS support for multimedia applications. The first version of the standard IEEE 802.162004 has been published in 2004 which describes the physical and MAC sublayers specifications for fixed wireless access systems supporting multiple services.The Second version of the standard IEEE 802.16e-2005 has been published in 2005 and contains numerous revisions, adds higher layer handovers between base stations, as well as support mobility at vehicular speeds.
The International Telecommunication Union standard X.902 refers to QoS as "a set of quality requirements on the collective behavior of one or more objects". A number of QoS parameters are used to describe the speed and reliability of data transmission. In wireless networks, including WiMAX, QoS support usually resides in the MAC sublayer because of the need to interact with radio resource management and physical layer dynamics. Some applications such as voice or video streaming over IP will have stringent delay and jitter constrains, while consuming little bandwidth; others, such as web browsing, require much more bandwidth but are more resilient to longer delays [3].

WiMAX allows to use the QoS requirements of certain application, like the maximum tolerated latency or the minimum throughput required to operate properly, The QoS parameters configurable depending on five different scheduling services; Unsolicited Grant Service (UGS), extended real-time Polling Service (ertPS), real-time Polling Service (rtPS), non-real-time Polling Service (nrtPS) and Best Effort (BE) [4].

The UGS support fixed sized data packets at a constant bit rate (CBR) such as E1/T1 lines that can sustain real-time data stream applications. This service provides guaranteed throughput, latency and jitter to the necessary levels as TDM services. The UGS is used mainly to support Constant Bit Rate (CBR) services found in voice applications such as voice over IP. rtPS support real-time service flow that generates variable sized data packets on a periodic interval with a guaranteed minimum rate and guaranteed delay. The mandatory service flow parameters that define this service are inclusive of minimum reserved traffic rate, maximum sustained traffic rate, maximum latency and request/transmission policy. The rtPS is used extensively in MPEG video conferencing and streaming. nrtPS is designed for non real-time traffics with no delay guaranteed. The delay tolerant data stream consists of variable sized data packets. The applications provided by this service are time insensitive and a minimum amount of bandwidth. This service is especially suitable for critical data application such as in File Transfer Protocol (FTP). The ertPS provides real-time applications which generate variable sized data packets periodically that require guaranteed data rate and delay with silence suppression. This service is only defined in IEEE 802.16e-2005. During the silent periods, no traffic is sent and no bandwidth is allocated. However, The BS poll SS to determine the end of the silent periods. BE provides support for data streams whereby no minimum service level guarantee is required. The mandatory service flow parameters that define this service include maximum sustained traffic rate, 
traffic priority and request/transmission policy. The BE supports data streams found in Hypertext Transport Protocol (HTTP) and electronic mail (email) [5].

Packet scheduling is the process of resolving contention for bandwidth. A scheduling algorithm has to determine the allocation of bandwidth between the users and their transmission order. One of the most important tasks of a scheduling scheme is to satisfy the QoS requirements of its users while efficiently utilizing the available bandwidth. The IEEE 802.16 standard does not specify which scheduling algorithm should be used. Vendors and operators have the choice among many existing scheduling techniques; they can also propose their own scheduling algorithms, robust scheduler for WiMAX give optimal performance is still an open research area [6].

The rest of this paper is organized as follows: section 2 presents the related works. Section 3 presents the signal to noise ratio model. Section 4 describes and discusses our scheduling algorithm. Section 5 presents the simulation and results. Finally, concluding remarks and some further future works are made in Section 6.

\section{RELATED WORKS}

Scheduling is one of the most important topics in WIMAX development. Currently in the literature, many scheduling algorithms are introduced. They could be classified into two main categories: channel-unaware and channel-aware schedulers. Basically, the channel-unaware schedulers use no information of the channel state condition in making the scheduling decision. The channel-aware schedulers take into account channel state and the channel quality variation between the subscriber station and the base station. Channelunaware schedulers generally assume error-free channel since it makes it easier to prove assurance of QoS. However, in wireless environment where there is a high variability of radio link such as signal attenuation, fading, interference and noise, the channel-awareness is important. Ideally, scheduler designers should take into account the channel condition in order to optimally and efficiently make the allocation decision [6].

Several research works have been conducted in order to provide QoS in IEEE 802.16 networks [7-16]:

In [7], a channel-unaware scheduling architecture for the IEEE 802.16 was presented. Different scheduling algorithm was implemented for the service flows, the UGS traffics are scheduled with First In First Out (FIFO), the rtPs traffics are scheduled with Earliest Deadline First (EDF), the nrtPs traffics are scheduled with Weighted fair queuing (WFQ) and Best Effort traffics are scheduled with Round Robin (RR). However, implementing different scheduling algorithm may increase the complexity and decrease the consistency of the system.

Ting et al. Proposed Random Early Detection (RED) based Deficit Fair Priority Queuing scheduler [8] for bandwidth allocation among the service classes of WiMAX networks. It uses dynamics Deficit Counters (DCs). First it transmits the rtPS packets and then the nrtPS packets will be transmitted. If there is no rtPS or nrtPS packet left, scheduler transmits BE packets. This algorithm ignores the channel quality and the low priority traffic will be suffered from starvation.

In [9], a channel-unaware scheduling algorithm was presented. It based on Round Robin but the channel quality was ignored. However, the main weakness of the study is treatment of time sensitive service flow and time insensitive service flow with the same manner.

New ideas were proposed in [10-11], it based on Deficit Round Robin (DRR) and Weighted Round Robin (WRR). The proposed algorithm used for the downlink and uplink schedulers, respectively. These algorithms are suitable for non real-time data services because they focus on the throughput guarantee of data flows and ignore the channel quality.

A Customized Deficit Round Robin (CDRR) was proposed in [12]. This scheduling algorithm is based on Modified Deficit Round Robin (MDRR) [13] with High Priority Queue (HPQ) and new Call Admission Control (CAC) framework, but it ignores the channel quality.

Wail and Mai proposed a Modified WRR Scheduling Algorithm for WiMAX Networks [14]. They integrate both WRR and Strict Priority (SP). The author ignores the channel quality which affects the overall performance.

Ali and Dimyati proposed new scheduling algorithms for Mobile WiMAX Systems called the Threshold based Cyclic Polling (TbCP) [15]. TbCP is a Priority Queue combine with threshold, but the channel quality was ignored.

Belghith and Nuaymi [16] discussed the maximum Signal to Interference Ratio (mSIR) which sorts the SSs bandwidth requests in descending order according to the received Signal to Noise Ratio (SNR) values and the BS serve the SSs in this order. Therefore, the mSIR has the heights throughput, but it does not guarantee fairness for small SNR to increase the capacity of the WiMAX system and it does not take into account the quality of service parameter like maximum latency. In addition, they proposed a new scheduling algorithm, namely, modified maximum Signal to Interference Ratio (mmSIR) which sorts the SSs in descending order according to the received signal to noise ratio (SNR) values and If the next SS to be served had a unicast opportunity in the next frame, the BS does not serve this SS. Otherwise, the BS serves the SSs having the highest SNR. The main drawback of mmSIR does not take into account the QoS parameters like maximum latency. So it serves real-time application and delay insensitive application with the same priority.

\section{SIGNAL TO NOISE RATIO MODEL}

The simulation scenario contains central base station surrounded by many subscriber stations. These subscriber stations are randomly deployed with random coordinates in the coverage area of the base station. At the start traffics initialized and admitted based on the resources needed by subscriber station. Each subscriber station has different Signal to Noise Ratio lead to change the Modulation and Coding Scheme (MCS) and affects the data rate.

\subsection{Modulation and Coding Scheme}

Wireless communication systems used digital modulation, which is to modulate an analog signal with a digital sequence in order to transport this digital sequence over a given medium. In wireless communication systems, random fluctuations prevent the continuous use of highly bandwidth-efficient modulation and the MCS depends upon the location of the SS.

The IEEE 802.16 standard supports many MCSs. In bad channel quality when SNR is low the IEEE 802.16 use low modulation technique which decrease the data rate and in vice versa. Four modulations techniques are supported by the IEEE 802.16 standard: Binary Phase Shift Keying (BPSK), 
Quadrature Phase Shift Keying (QPSK), 16-Quadrature Amplitude Modulation (16-QAM) and 64-Quadrature Amplitude Modulation (64-QAM).

Table 1 shows the Modulation and coding rate, received signal to noise ratio, the received uplink and downlink data rate with frame duration $5 \mathrm{~ms}$, channel bandwidth $10 \mathrm{MHz}$ and TDD mode. As shown in the table when SNR increase the uplink and downlink data rate increase and when the SNR decrease the uplink and downlink data rate decrease and this is due to the change of modulation technique.

Table 1. Ieee 802.16e MCS and SNR [17-18]

\begin{tabular}{|c|c|c|c|c|}
\hline MCS & $\begin{array}{c}\text { bits / } \\
\text { symbol }\end{array}$ & $\begin{array}{c}\text { Receiver } \\
\text { SNR(db) }\end{array}$ & $\begin{array}{c}\text { Uplink } \\
\text { Rate, Mbps }\end{array}$ & $\begin{array}{c}\text { Uplink } \\
\text { Rate, Mbps }\end{array}$ \\
\hline QPSK 1/2 & 2 & 5 & 6.34 & 4.70 \\
\hline QPSK 3/4 & 2 & 8 & 9.50 & 7.06 \\
\hline 16-QAM 1/2 & 4 & 10.5 & 12.67 & 9.41 \\
\hline 16-QAM 3/4 & 4 & 14 & 19.01 & 14.11 \\
\hline 64-QAM 1/2 & 6 & 16 & 19.01 & 14.11 \\
\hline 64-QAM 2/3 & 6 & 18 & 25.34 & 18.82 \\
\hline 64-QAM 3/4 & 6 & 20 & 28.51 & 21.17 \\
\hline 64-QAM 5/6 & 6 & 22 & 31.68 & 21.17 \\
\hline
\end{tabular}

\subsection{Channel Model}

A channel model is an important factor which significantly influences the decision of scheduling algorithm in the IEEE 802.16 systems. The channel model that used with the simulation with COST-231 Hata path loss is as follows [19]:

$$
\begin{aligned}
& S N R=P_{t}-P_{L}-N_{\text {noise }} \\
& P_{t}=43+G_{t}+G_{r} \\
& N_{\text {noise }}=-174+10 \log _{10}(B W)+N F
\end{aligned}
$$

Where $\mathrm{P}_{\mathrm{t}}$ is transmitted power, $\mathrm{P}_{\mathrm{L}}$ is the COST-231 Hata path loss, $G_{t}$ is transmitter antenna gain, $G_{r}$ is receiver antenna gain, BW is the system bandwidth and NF is the noise figure.

$$
\begin{gathered}
P_{L}=46.3+33.9 \log _{10}(f)-13.82 \log _{10}\left(h_{B}\right)-A\left(h_{R}\right) \\
+\left[44.9-6.55 \log _{10}\left(h_{B}\right)\right] \log _{10} d+C
\end{gathered}
$$

Where $\mathrm{C}$ is a correction factor and equal to $3, \mathrm{f}$ is the frequency of transmission, $h_{B}$ is the base station antenna effective height, $d$ is the link distance, $h_{R}$ is the subscriber station antenna effective height, $A\left(h_{R}\right)$ is the subscriber station Antenna height correction factor.

\section{PROPOSED SOLUTION}

In this work, we propose a new scheduling algorithm, namely, Deadline maximum Signal to Interference Ratio (DmSIR) and it is derived from mSIR. The mSIR sort the SSs bandwidth requests in descending order according to the received SNR values and the BS serve the SSs in this order. Through this process the mSIR working to increase the bandwidth utilization and this occurs via using the height Modulation and Coding Scheme which leads to increase the number of bits per an OFDM symbol. However, the mSIR does not take into account the service flow types and treat all service flows in the same way. The DmSIR algorithm scheduled packet based on two criteria; one is the link quality, which is use in chosen modulation and deadline for each packet. The DmSIR Depends of two queue the Idle Queue (IQ) and the Deadline Queue (DQ).

In downlink bandwidth allocation phase the DmSIR moves and sorts the packets in the Idle Queue based on Signal to
SNR, any packet could meet deadline in next frame it move from the Idle Queue to Deadline Queue. In this algorithm deadline is defined as:

deadline $=\left\lfloor\frac{\mathrm{L}}{\mathrm{F}}\right\rfloor\left\{\begin{array}{l}<1 \text { deadline expire must be dropped } \\ =1 \text { meet deadline next frame } \\ >1 \text { still time to meet deadline }\end{array}\right.$

Where $\mathrm{L}$ is the maximum latency for the service flow and $\mathrm{F}$ represents the frame duration and the frame duration value used in the simulation is $5 \mathrm{~ms}$. The frame contains a number of slots, the modulation and coding scheme decides the number of bits carried in slot which described in this equation $[17,20]$ :

$$
\text { Slot Size }=48 \times \mathrm{MB} \times \mathrm{CR}
$$

Where MB is the modulation bit, $\mathrm{CR}$ is the coding rate and both depend on the modulation and coding scheme. The number of slot required by packet could be determined through divide packet size by slot size.

The allocation procedure is done as follows:

i) Calculates the packets deadline using the previous formula. And then move packets which meet deadline next frame From the Idle Queue to the Deadline Queue and drop packets that ended their deadline.

ii) If the Deadline Queue does not empty the packets in this queue it will be served first and if there a reminding slot the Idle Queue will be served until all slots fills up.

iii) Otherwise if the Deadline Queue is empty the Idle Queue will be served.

In uplink bandwidth grant phase the DmSIR works as the same way of downlink bandwidth allocation. In both downlink and uplink bandwidth allocations the DmSIR use the backlog SNR. After allocating bandwidth using the DmSIR scheduler, the DL and UL MAPs will be generated and sent to notify the SSs of the transmission and receiving opportunities.

Fig1 show the pseudo code of the DmSIR scheduling algorithm. 

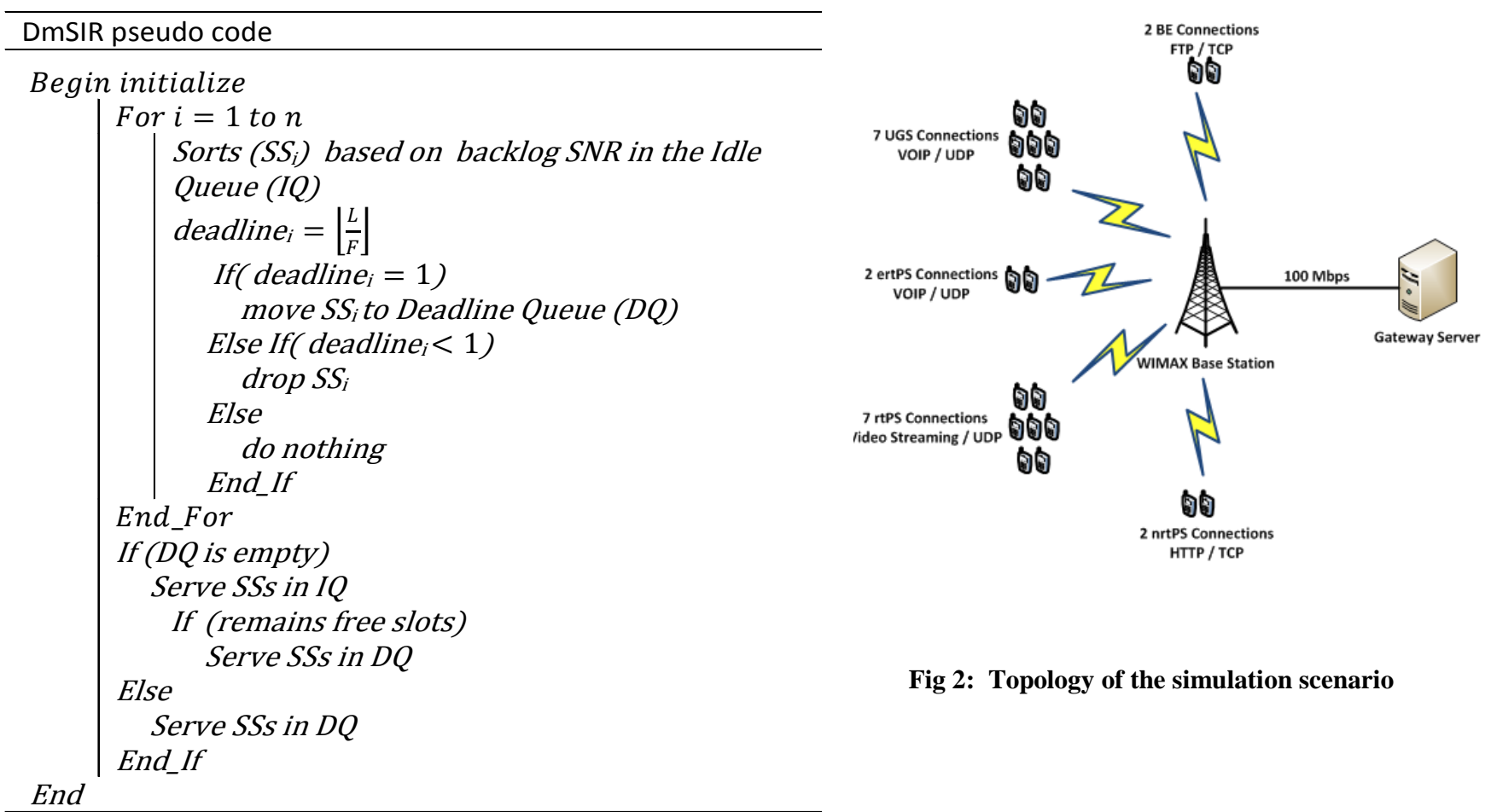

Fig 1: DmSIR pseudo code

\section{SIMULATION AND RESULTS}

The DmSIR scheduling algorithm is evaluated under the ns2 [21] simulation environment, using modified version of WiMAX module developed by Telecom Bretagne [22] and the OFDM physical layer was extended to support OFDMA. The topology of the simulation scenario is presented in Fig 2; the scenario consists of a single sector with single base station and twenty subscriber stations distributed randomly with Random Waypoint mobility model. The subscriber stations establish seven UGS, two ertPS, seven rtPS, two nrtPS and two BE traffic flows.

The subscriber stations are randomly deployed with random coordinates in the coverage area of the BS. The BS connected with gateway server (Sink Node) through 100Mbps full duplex link. The IEEE 802.16 has a set of QoS parameters such as The Minimum Reserved Traffic Rate (MRTR), Maximum Sustained Traffic Rate (MSTR), Maximum latency (ML), Tolerated Packet Loss (TPL) and Packet Size in bytes (PS).These QoS parameters vary according to the traffic flows, Table 2 shows the QoS parameters according to the traffic flows used in the simulation.

The most of the WiMAX parameters in the simulation are based on the WiMAX Forum Mobile System profile [23]. These parameters value are represented in Table 3.

Table 2. QoS parameters for different traffic classes

\begin{tabular}{|c|c|c|c|c|c|}
\hline Parameter & UGS & ertPS & rtPS & nrtPS & BE \\
\hline MRTR (kbps) & 10 & 8 & 64 & 128 & - \\
\hline MSTR (kbps) & 64 & 128 & 300 & 512 & 1024 \\
\hline ML (ms) & 50 & 100 & 150 & 500 & - \\
\hline TPL (\%) & 10 & 30 & 3 & - & - \\
\hline PS (bytes) & 40 & 80 & $150-300$ & 1500 & 1500 \\
\hline
\end{tabular}

Fig 2: Topology of the simulation scenario \begin{tabular}{l|r} 
Table 3. Simulation parameters \\
\hline Values
\end{tabular}

\begin{tabular}{|c|c|}
\hline Parameter & Values \\
\hline Topology & Single BS with multiple MSs \\
\hline Spectrum & $5.0 \mathrm{GHz}$ \\
\hline Physical Layer & OFDMA - TDD \\
\hline System Bandwidth & $10 \mathrm{MHz}$ \\
\hline Sampling factor & $28 / 25$ \\
\hline FFT size (NFFT) & 1024 \\
\hline Propagation model & COST-231 Hata Model \\
\hline Antenna model & Omni antenna \\
\hline Link adaptation & Enabled \\
\hline BS Antenna Height $\left(\mathrm{h}_{\mathrm{B}}\right)$ & 32 meters \\
\hline SS Antenna Height $\left(h_{R}\right)$ & 1.5 meters \\
\hline BS Antenna Gain $\left(G_{t}\right)$ & $16 \mathrm{dBi}$ \\
\hline MS Antenna Gain $\left(\mathrm{G}_{\mathrm{r}}\right)$ & $0 \mathrm{dBi}$ \\
\hline $\begin{array}{l}\text { BS Maximum Power Amplifier } \\
\text { Power }\end{array}$ & $43 \mathrm{dBm}$ \\
\hline $\begin{array}{l}\text { Mobile Terminal Maximum PA } \\
\text { Power }\end{array}$ & $23 \mathrm{dBm}$ \\
\hline BS Noise Figure (NF) & $5 \mathrm{~dB}$ \\
\hline SS Noise Figure (NF) & $7 \mathrm{~dB}$ \\
\hline Frame duration & $5 \mathrm{~ms}$ \\
\hline Downlink: Uplink symbols & $26: 21$ \\
\hline TTG+RTG & 1.6 symbol \\
\hline Permutation & PUSC \\
\hline No. of OFDMA symbols /frame & $47+1$ Preamble \\
\hline OFDMA Symbol Duration & 102.9 us \\
\hline Max. Packet Size & 1500 bytes \\
\hline Simulation duration & $100 \mathrm{~s}$ \\
\hline
\end{tabular}

One of the main objectives of this paper is to analyze the performance of the DmSIR scheduling algorithm. To analyze the performance of the DmSIR three performance metrics are used:

i) Number of deliver packets: is used to measure number of deliver packets by the network scenario as a function traffic load. 
ii) Average end to end delay: is the mean value between the values that represent the time difference between the instant of the reception of the final bit in the packet and the instant of the sending of the first bit in the packet by the application as a function of traffic load.

$$
\mathrm{AD}=\frac{\sum_{\mathrm{i}=1}^{\mathrm{n}}\left(\mathrm{PR}_{\mathrm{i}}-\mathrm{PS}_{\mathrm{i}}\right)}{\mathrm{n}}
$$

Where AD is the average end to end delay, PR is the packet reception time, PS is the packet sending time and $\mathrm{n}$ is the total number of packets.

iii) Fairness index: is the arithmetic mean of served subscriber stations per frame as a function of traffic load.

$$
F I=\frac{1}{q} \times \sum_{j=1}^{q} z_{i}
$$

Where FI is the fairness index, $z_{i}$ is the of served subscriber stations per frame, $\mathrm{q}$ is the total number of frames.

Fig 3 shows the number of delivered data packets as a function of the traffic load submitted to the network scenario. This figure shows low packets deliver of both the mSIR and the mmSIR scheduling algorithm due the ignorance of maximum latency (delay) of the packets. On the other hand, the DmSIR scheduler gives the radio resources to the SS that have the highest SIR until one or more SS meets their deadline, in this case they will be served first. Thus the DmSIR meet the maximum latency of the most SS.

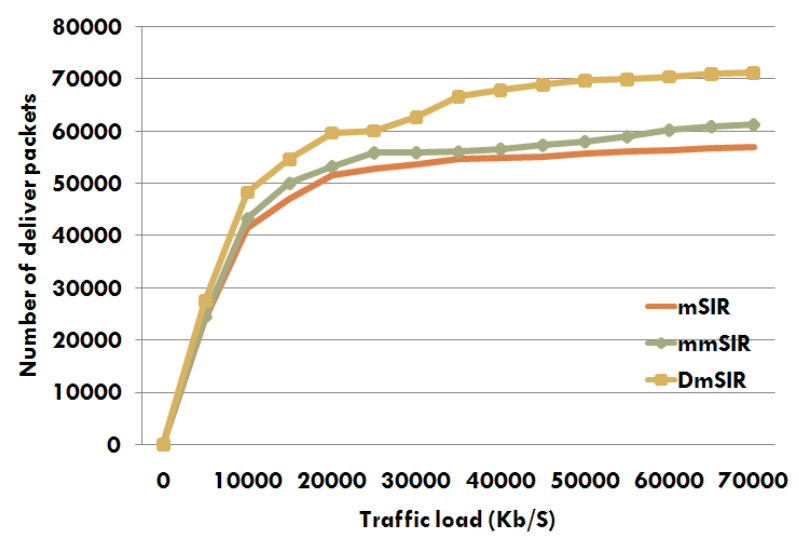

Fig 3: Number of delivered packets versus traffic load

Fig 4, shows the average end to end delay as a function of the traffic load submitted to the network scenario for the three scheduling algorithms. It is important to note that both mSIR and mmSIR scheduling algorithm ignore deadline of packets; thus they have higher latency in both way uplink and downlink. The simulation show the mSIR have much more average end to end delay compared to mmSIR scheduling algorithm. Also figure 4 shows the DmSIR scheduler provides less average end to end delay. This is mainly due to the consideration of maximum latency of packet and that decrease the packets retransmission.

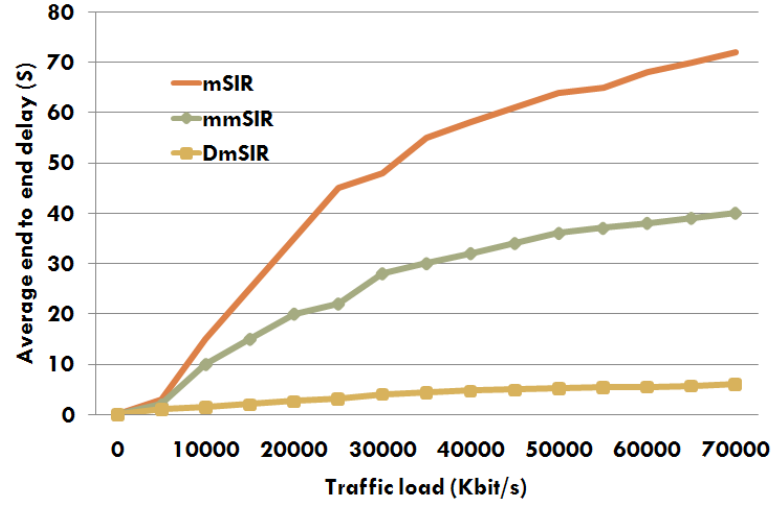

Fig 4: Average end to end delay versus traffic load

The fairness index as a function of traffic load is represented in Fig 5 .The DmSIR scheduler serves more SSs per frame because it always serves the SSs which close meet their deadline and these SSs usually have small packets size as shown in table II the real-time traffics (UGS, ertPS and rtPS) have small packet size. In this network scenario both mSIR and mmSIR scheduler show low fairness index does not exceed 4 , thus because both mSIR and mmSIR seeking to packets that have heights SIR and ignore real-time application packets which have small packets size.

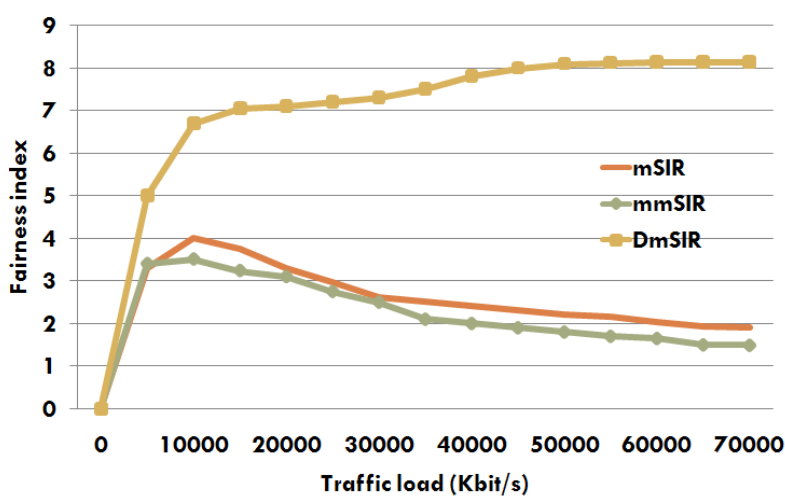

Fig 5: Fairness index versus traffic load.

\section{CONCLUSION}

The IEEE 802.16 standard for broadband wireless access is a low cost solution for Internet access in metropolitan and rural areas. Although it defines five service levels to support realtime and bandwidth demanding applications, scheduling mechanisms are not specified in the standard. In this paper, we presented a new scheduling algorithm for IEEE 802.16. In order to make scheduling decision, the proposed scheduler use both link quality and deadline. With the use of ns 2 simulator we compared the DmSIR with mSIR and mmSIR. Simulation Results show that the proposed approach gain more delivering packet and decrease the average the end to end delay and doubling the fairness index.

Finally, we would like to mention some future research directions. We will work on integrate the Call Admission Control (CAC) with DmSIR and the possibility of adding separate queue for each service flow and its effect on the performance.

\section{REFERENCES}

[1] IEEE 802.16-2004, "IEEE standard for Local and Metropolitan Area Networks - Part 16: Air interface for 
Fixed Broadband Wireless Access systems," October 2004.

[2] IEEE 802.16e-2005, "IEEE Standard for Local and Metropolitan Area Networks - Part 16: Air interface for Fixed Broadband Wireless Access systems Amendment 2: Physical and Medium Access Control layers for combined fixed and mobile operation in licensed bands and Corrigendum 1," February 2006.

[3] ITU-T Recommendation X.902, "Information technology - Open Distributed Processing - Reference Model : Foundations," November 1995.

[4] Y. A. Şekercioğlua, M. Ivanovicha, and A. Yeğin, "A Survey of MAC based QoS Implementations for WiMAX Networks," Computer Networks (Elsevier), Vol. 53, no. 14, pp. 2517-2536, Sept. 2009.

[5] Nuaymi L., "WiMAX: Technology for Broadband Wireless Access," John Wiley \& Sons, 2007.

[6] C. So-In, R. Jain, and A. Tamimi, "Scheduling in IEEE 802.16e mobile WiMAX networks: key issues and a survey," IEEE J. Sel. Areas Commun., vol. 27, no. 2, pp. $156-171,2009$.

[7] K. Wongthavarawat and A. Ganz, "Packet Scheduling for QoS Support in IEEE 802.16 Broadband Wireless Access Systems", International Journal of Communication Systems, Vol. 16, 2003, pp81-96.

[8] Po-Chun Ting, Chia-Yu Yu, and Naveen Chilamkurti, "A Proposed RED-based Scheduling Scheme for QoS in WiMAX Networks," IEEE International Symposium on Wireless Pervasive Computing (ISWPC), February 2009.

[9] C. F. Ball, F. Treml, K. Ivanov, and E. Humburg, "Performance evaluation of IEEE 802.16 WIMAX with fixed and mobile subscribers in tight reuse," European Transactions on Telecommunications, Vol. 17, pp.20321., March 2006.

[10] C. Cicconetti, et al., "Quality of service support in IEEE 802.16 networks," IEEE Network Magazine, Vol. 20, Issue 2, pp. 50-55, 2006.

[11] C. Cicconetti, L. Lenzini, and E. Mingozzi, "Performance evaluation of the IEEE 802.16 MAC for QoS support," IEEE Transactions on Mobile Computing, Vol. 6, no. 1, pp. 26-38, January 2007.
[12] E. Laias, I. Awan, and P. M. L. Chan, "Fair and Latency Aware Uplink Scheduler in IEEE 802.16 Using Customized Deficit Round Robin," Advanced Information Networking and Applications Workshops, pp. pp.425-432, May 2009.

[13] Cisco Systems, "Understanding and Configuring MDRR/WRED on the Cisco 12000 Series Internet Router", (http://www.cisco.com/warp /public/63/mdrr_wred_overview.html).

[14] W. Mardini, M.A. Alfool, "Modified WRR Scheduling Algorithm for WiMAX Networks," Network Protocols and Algorithms journal, Vol. 3, No.2, 2011.

[15] D. M. Ali and K. Dimyati, "Threshold based Cyclic Polling (TbCP): An Uplink Scheduling Algorithm for Mobile WiMAX Systems," International Journal of Information and Electronics Engineering, Vol. 1, No. 1, July 2011.

[16] A. Belghith and L. Nuaymi, "Comparison of WiMAX scheduling algorithms and proposals for the rtPS QoS class," 14th European Wireless Conference, 22-25 June, 2008.

[17] WiMAX Forum, "Mobile WiMAX: A technical overview and performance evaluation," June 2006.

[18] Z. Abate, "WiMax RF systems engineering", ARTECH HOUSE, Boston, 2009, pp.68-73.

[19] Peng Wu,Tsung Tsa,Yung-Cheng Kao,Jenq-Neng Hwang, and Chung-Nan Lee, "An NS2 Simulation Module for Multicast and OFDMA of IEEE 802.16e Mobile WiMAX," unpublished.

[20] Lin, Y. N., Lin, Y. D., Lai, Y. C. and Wu, C. W, "Highest Urgency Firs (HUF): A latency and modulation aware bandwidth allocation algorithm for WiMAX base stations," Computer Communications, vol. 32, pp. 332342, 2009.

[21] The Network Simulator (NS2), http://www.isi.edu/nsnam/ns/).

[22] A. Belghith and L. Nuaymi, "Design and implementation of a QoS-included WiMAX module for NS-2 simulator," SIMUTools 2008, France, March 2008.

[23] WiMAX Forum, "WiMAX System Evaluation Methodology "WiMAX," (www.wimaxforum.org/technology/documents/) 